\title{
Knowledge and perception on the transmission and control of SARS-COV-2 infection among allied radiation medicine professionals in Ghana
}

\author{
Francis Hasford ${ }^{1,2}$. Edem Kwabla Sosu ${ }^{1,2}$. Adolf Kofi Awua ${ }^{1,2}$. Prince Rockson ${ }^{3}$. Emmanuel Nii Boye Hammond ${ }^{1,4,5}$
}

Received: 5 September 2020 / Accepted: 2 November 2020 / Published online: 27 November 2020

( ) IUPESM and Springer-Verlag GmbH Germany, part of Springer Nature 2020

\begin{abstract}
Knowledge and perception on transmission and control of SARS-COV-2 infection are key to preventing outbreak of the disease in healthcare settings and in dealing with the COVID-19 pandemic. This study assessed the level of knowledge on SARS-COV-2 infection prevention, transmission and symptoms of COVID-19, as well as perceptions regarding prevention of SARS-COV-2 infection among allied radiation medicine professionals. Cross-sectional descriptive survey was carried out among 145 radiation medicine professionals in Ghana using facts on COVID-19 as presented on the website of the World Health Organization and data was analyzed based on weighted average indices. Overall, the extent of knowledge among allied radiation medicine professionals on the symptoms of COVID-19, transmission and control of SARS-COV-2 infection in radiation medicine facilities were all adequate, with weighted average indices of 3.8, 4.1 and 4.4 respectively. However, overall perception of the respondents regarding the use of radiation medicine procedures in management of COVID-19 was diverse, with weighted index of 3.5. The facts about COVID-19 that were identified to be most known were shortness of breath being a serious symptom of the disease and fever being a common symptom. The extent of knowledge on the fact that "SARS-COV-2 infection can be transmitted through small droplets from the nose or mouth of an infected person" was almost excellent, with weighted average index of 4.9. Also, the thinking that provision of hand washing and sanitizing facilities is a measure for controlling the infection was almost perfectly shared among the respondents. Computed tomography was perceived by majority of the respondents as the most preferred imaging modality for screening patients for COVID-19. The study shows that some aspects of the awareness of radiation medicine professionals on COVID-19 pandemic are adequate and others need critical improvement to help reduce spread of the disease.
\end{abstract}

Keywords COVID-19 $\cdot$ SARS-COV-2 $\cdot$ Radiation medicine $\cdot$ Knowledge and perception $\cdot$ Ghana

\section{Background}

This article is part of the COVID-19 Health Technology: Design, Regulation, Management, Assessment

Francis Hasford

haspee@yahoo.co.uk

1 School of Nuclear and Allied Sciences, University of Ghana, Accra, Ghana

2 Radiological and Medical Sciences Research Institute, Ghana Atomic Energy Commission, Accra, Ghana

3 University of Health and Allied Sciences, Ho, Ghana

4 GAEC Hospital, Ghana Atomic Energy Commission, Accra, Ghana

5 National Centre for Radiotherapy and Nuclear Medicine, Korle-Bu Teaching Hospital, Accra, Ghana
Coronavirus disease 2019 (COVID-19) is a deadly disease caused by the novel coronavirus [1]. Coronaviruses in human beings cause respiratory, gastrointestinal and central nervous system diseases [2]. In December 2019, the seventh coronavirus known to infect humans was found in China (Wuhan city, Hubei Province) [3] and on 12 January 2020, the World Health Organization (WHO) announced the temporarily named nCoV-2019, now called SARS-CoV-2. The WHO declared the disease a global pandemic in March 2020 [4, 5]. Worldwide, 25.7 million people have been infected with COVID-19 since it was first identified, with about 850,000 deaths as at September 1, 2020. Presently, there are 6.8 million active cases of the disease across the world, of which $99 \%$ are of mild conditions [6]. Ghana announced 
the presence of the disease in the country (through vertical transmission) by confirming the first two COVID-19 cases on March 12, 2020. As at September 1, 2020, Ghana has recorded 44,298 confirmed cases with 276 deaths [7].

Reverse-transcription polymerase chain reaction (RTPCR) assay has been the most preferred method for diagnosis of COVID-19 infections worldwide. In some instances, computed tomography (CT) and X-ray imaging of the thorax have been employed for diagnosing COVID-19 patients who manifest conditions such as difficulty in breathing. Also the imaging modalities have been employed for some suspected COVID-19 cases due to regional preferences, lack of availability of PCR assays, and false-negative PCR assays, as well as for monitoring of disease progression, complications, and treatment response [8].

Knowledge and perception of healthcare professionals on transmission and control of SARS-COV-2 infection are essential to avert outbreak of the disease in healthcare settings. With these in mind, this survey was designed using the facts on COVID-19 as stated by the WHO [9], to assess the levels of awareness of radiation medicine professionals about the COVID-19 pandemic as part of the means to prevent the outbreak in radiation medicine facilities. Specifically, the study determined the level of knowledge on the SARS-COV-2 infection prevention, transmission and symptoms of COVID-19 as well as perceptions regarding prevention of SARS-COV-2 infection in relation to the radiation medicine practices in radiological health facilities. Findings of the survey would confirm the need or otherwise for a tailored and specific educational and other interventions to reduce the risk of the transmission of SARS-COV-2 in radiation medicine facilities. Additionally, it will provide relevant information on the appropriate translation of the general knowledge on infection prevention to practice-related preventive activities in the practise of radiation medicine. Using the facts on COVID-19 as stated by the WHO provides standardized information that will be useful for global comparison and adoption.

\section{Methods}

\subsection{Study design and population}

This cross-sectional descriptive survey was carried among radiation medicine professionals who were reached on online, using the professional groups' WhatsApp platforms. The WhatsApp platforms were purposively selected to include all the related professions that work in radiation medicine. Any professional member in active service who was on any of the professional WhatsApp platforms was included in the study.

\subsection{Study tool}

The survey tool was designed and developed de novo, using facts on COVID-19 as presented on the WHO's website as at $17^{\text {th }}$ April, 2020. Additionally, few relevant misconceptions and myths were used in the development of the survey tool. The tool consisted of nine symptoms considered to be either common, less common or serious. It also consisted of six facts on the transmission of the SARS-COV-2 infection. Furthermore, and of most importance was the inclusion of 12 facts related to the prevention/control of the infection at a radiation medicine facility. Lastly, five facts about the use of radiation medicine procedures and technologies in the fight against COVID19 were included. The tool was a structured questionnaire which was self-administered.

\subsection{Ethical considerations}

This minimal risk-survey was designed in line with ethical principles related to the protection of research participants. Given that the target population of the study were persons who have had higher than secondary education, a selfadministered information sheet (provided on the first page of the questionnaire) informed the participant of the purpose of the study, the risk of participation, what was required of a respondent, and the lack of direct benefit to respondents. Persons who were willing to participate in the survey were to indicate so by ticking a box on the form, which subsequently activated the next steps on the Google Form. Confidentiality of the data was ensured by not collecting information that will lead to identification of the respondent. Access to the data on the server was restricted by a two-way pass code.

\subsection{Data management and analysis}

Given that a Google form was used for data collection, data was directly entered into Microsoft Excel, 2013 version (Microsoft Corp., USA). All responses on the Likert scale were coded (weighted) on a scale of 1 to 5 , where 1 and 2 (average of 1.0 to 2.5) indicated inappropriate level of the measure of interest, 3 (2.6 to 3.5) indicated limited level of the measure of interest (such as lack of knowledge) and 4 and 5 (3.6 to $4.5 ; 4.6$ to 5.0) indicated adequate and appropriate level of the measure of interest. The Weighted Average Index (WAI) of the responses to each question was determined. Again, the WAI of the responses of each respondent to question related to each measure were determined as the composite level of each measure. The average of these represented the composite level of each measure for the survey; these were the primary outcomes. Additionally, 
the proportion of the respondents (distribution) on the scale was also determined and presented as a bar chart.

\section{Results}

\subsection{Participants' characteristics}

The distribution of the characteristics of the 145 respondents showed that most of them were male $(77.9 \%)$ and within the age groups of $30-39$ years $(62.1 \%)$. The commonest highest attained educational level of the participants were Bachelor's (39.1\%) and Master's degrees (42.1\%). Given that the survey was targeted at a professional group, the distribution showed that diagnostic radiographers were the majority $(65.5 \%)$; however, all the closely related professionals were represented (Table 1). Again, the respondents were from all types of health facilities, but respondents from Tertiary healthcare public facilities were the majority $(37.9 \%)$.

\subsection{Knowledge on COVID-19}

The results as presented in Table 2 shows that overall, the extent of knowledge on the symptoms of COVID-19 among the respondents was adequate (WAI of 3.8). The symptoms of COVID-19 that was most known, and at the appropriate level among the respondents were; Shortness of breath as a serious symptom, and Fever is a common symptom, with WAI of 4.7 and 4.6 (on a scale of 1 to 5) respectively. Table 2 presents the WAIs of the remaining symptoms; three were at adequate level, and four were at limited level.

Again, the overall or composite level of knowledge among the respondents, this time on the transmission of the causative virus of COVID-19, was adequate (WAI =4.1; Table 3). Specifically, the extent of knowledge on the fact that "SARS-COV-2 (COVID-19) infection can be transmitted through small droplets from the nose or mouth of an infected person" among the respondents was almost excellent, with a WAI of 4.9. Among the remaining five facts, as presented in Table 3 with their respective WAI, four were at an adequate level, while the fact "The risk of transmission of SARS-COV-2 (COVID-19) through faeces is very high" was limited (WAI $=3.5)$.

\subsection{Perceptions related to COVID-19 and radiation medicine procedures}

The overall thinking regarding the 12 measures of control of the infection of SARS-COV-2 in a radiation medicine facility was determined to be adequate for preventing the
Table 1 Distribution of participants' characteristics

\begin{tabular}{|c|c|c|c|}
\hline Characteristics & Categories & $\begin{array}{l}\text { Frequency } \\
(\mathrm{N}=145)\end{array}$ & $\%$ of tota \\
\hline \multirow[t]{2}{*}{ Gender } & Female & 32 & 22.1 \\
\hline & Male & 113 & 77.9 \\
\hline \multirow[t]{5}{*}{ Age (years) } & $20-29$ & 7 & 4.8 \\
\hline & $30-39$ & 90 & 62.1 \\
\hline & $40-49$ & 41 & 28.3 \\
\hline & $50-59$ & 6 & 4.1 \\
\hline & $>60$ & 1 & 0.7 \\
\hline \multirow{5}{*}{$\begin{array}{l}\text { Highest attained Edu- } \\
\text { cational level }\end{array}$} & Certificate & 1 & 0.7 \\
\hline & Diploma & 19 & 13.1 \\
\hline & Bachelor's degree (BSc) & 57 & 39.3 \\
\hline & Master's degree (MSc/MPhil) & 61 & 42.1 \\
\hline & Doctorate degree $(\mathrm{PhD})$ & 7 & 4.8 \\
\hline \multirow[t]{5}{*}{ Occupation } & Diagnostic Radiographer & 95 & 65.5 \\
\hline & Medical Physicist & 26 & 17.9 \\
\hline & Radiological Technician & 16 & 11.0 \\
\hline & Sonographer & 5 & 3.4 \\
\hline & Therapy Radiographer & 3 & 2.1 \\
\hline \multirow[t]{6}{*}{ Facility } & Private healthcare facility & 18 & 12.4 \\
\hline & Public Hospital—Primary healthcare facility & 17 & 11.7 \\
\hline & Public Hospital—Secondary healthcare facility & 23 & 15.9 \\
\hline & Public Hospital—Tertiary healthcare facility & 55 & 37.9 \\
\hline & Quasi-Government healthcare facility & 18 & 12.4 \\
\hline & Research / Academic Institution & 14 & 9.7 \\
\hline
\end{tabular}


Table 2 Level of knowledge on the symptoms of COVID-19

\begin{tabular}{lll}
\hline Facts on COVID-19 Symptoms & WAI & Inference \\
\hline Shortness of breath is a serious symptom of COVID-19 & 4.7 & Appropriate \\
Fever is a common symptom of COVID-19 & 4.6 & Appropriate \\
Dry cough is a common symptom of COVID-19 & 4.4 & Adequate \\
Chest pain is a serious symptom of COVID-19 & 4.1 & Adequate \\
Tiredness is a common symptom of COVID-19 & 4.0 & Adequate \\
Loss of taste is a less common symptom of COVID-19 & 3.1 & Limited \\
Headache is a less common symptom of COVID-19 & 3.1 & Limited \\
Sore throat is a less common symptom of COVID-19 & 3.1 & Limited \\
Loss of speech and movement are serious symptoms of COVID-19 & 2.8 & Limited \\
Overall / Composite score & 3.8 & Adequate \\
\hline
\end{tabular}

$\mathrm{WAI}=$ weighted average index on a scale of 1 to 5 spread of COVID-19 in such facilities (Table 4). Specifically, the thinking that "Hand washing and sanitizing facilities are required to be made available in a radiation medicine facility" was almost perfectly shared among the respondents $(\mathrm{WAI}=4.9)$. Table 4 shows that the extent of the perception on eight other control measures were appropriate, with WAI ranging between 4.6 and 4.8. The extent of the perception on the control measure "After scanning or treatment of a suspected COVID-19 patient in a radiation medicine department, the equipment has to be completely avoided for up to $1 \mathrm{~h}$ " was the least perceived procedure in the control of spread of COVID-19 (WAI =2.9) on the control of infection at such radiation medicine facilities.

A further analysis of the overall perception of the respondents regarding the use of radiation medicine procedures in management of COVID-19 was diverse (WAI 3.5; Table 5). The plurality of perception of the respondents on two of the five facts showed positive consensus (3.6 to 4.2) and the remaining three were diverse (WAI of 2.6 to 3.5). The most perceived fact about radiation medicine procedures (highest positive consensus) was that computed tomography (CT) is the most preferred imaging modality for screening patients for COVID-19 $(\mathrm{WAI}=4.2)$.

\section{Discussion}

In order to contribute to the fight against the COVID-19 pandemic in Ghana, and provide insights that are useful for other regions of the world, in keeping with the ethical principle of solidarity, this survey worked at assessing the level of awareness of radiation medicine professionals to prevent the transmission of SARS-COV-2 in radiation medicine facilities.

The effectiveness of the sampling approach employed (WhatsApp platform of professional groups) at ensuring appropriate internal and external validities of the survey was confirmed by having a similar distribution of the categories of the professions as is in the professional association's register (Table 1). Specifically, the ratio of males to females was about 3:1. However, the category of professions were only limited to the five indicated in Table 1 and the proportions of the categories may not reflect the general distribution.
Table 3 Level of knowledge on transmission of SARS-COV-2

\begin{tabular}{lcc}
\hline Facts on Transmission & WAI & Inference \\
\hline $\begin{array}{l}\text { SARS-COV-2 (COVID-19) infection can be transmitted through small droplets from } \\
\text { the nose or mouth of an infected person }\end{array}$ & 4.9 & Appropriate \\
Cleaning and draping of radiation emitting equipment is a necessary step to limit & 4.4 & Adequate \\
SARS-COV-2 (COVID-19) transmission & 4.1 & Adequate \\
SARS-COV-2 (COVID-19) infection spreads through airborne transmission & 4.1 & Adequate \\
COVID-19 patients are most infectious when they show symptoms & 3.9 & Adequate \\
SARS-COV-2 (COVID-19) cannot be transmitted through cooked food & 3.5 & Limited \\
The risk of transmission of SARS-COV-2 (COVID-19) through faeces is very high & 4.1 & Adequate \\
Overall / Composite score & &
\end{tabular}

$\mathrm{WAI}=$ weighted average index on a scale of 1 to 5 
Table 4 Perception on the control of SARS-COV-2 infection in radiation medicine facilities

\begin{tabular}{|c|c|c|}
\hline Facts on Infection Control & WAI & Inference \\
\hline Hand washing and sanitizing facilities are required to be made available in a radiation medicine facility & 4.9 & Appropriate \\
\hline $\begin{array}{l}\text { Decontamination is important for infection control in a radiation medicine facility after imaging / treating suspected } \\
\text { COVID-19 patients }\end{array}$ & 4.8 & Appropriate \\
\hline $\begin{array}{l}\text { Number of accompanying patient relatives to radiation medicine facilities are recommended to be restricted to avoid conges- } \\
\text { tion }\end{array}$ & 4.8 & Appropriate \\
\hline Both patients and staff are required to be screened (temperature check) before entry into a radiation medicine facility & 4.8 & Appropriate \\
\hline A good practice in radiation medicine facilities during COVID-19 pandemic is to place staff on shift system & 4.7 & Appropriate \\
\hline Radiation medicine professionals are required to undergo training on the safe use personal protective equipment (PPEs) & 4.7 & Appropriate \\
\hline $\begin{array}{l}\text { Maintenance of air flow is important for infection control in a radiation medicine facility after imaging / treating suspected } \\
\text { COVID-19 patients }\end{array}$ & 4.6 & Appropriate \\
\hline Wearing of face mask is effective against SARS-COV-2 infection & 4.6 & Appropriate \\
\hline Symptoms of COVID-19 in infected persons are observed mostly between $2-14$ days & 4.6 & Appropriate \\
\hline Ideal distance to be maintained from an infected person with COVID-19 is $1 \mathrm{~m}$ ( 3 feet) & 4.0 & Adequate \\
\hline $\begin{array}{l}\text { It is advisable to disinfect radiation medicine equipment after each working day, only when such equipment was used on } \\
\text { suspected COVID-19 patient(s) }\end{array}$ & 3.3 & Limiting \\
\hline $\begin{array}{l}\text { After scanning or treatment of a suspected COVID-19 patient in a radiation medicine department, the equipment has to be } \\
\text { completely avoided for up to } 1 \mathrm{~h}\end{array}$ & 2.9 & Limiting \\
\hline Overall / Composite Score & 4.4 & Adequate \\
\hline
\end{tabular}

$\mathrm{WAI}=$ weighted average index on a scale of 1 to 5

The selected radiation medicine professionals (diagnostic radiographers, medical physicists, radiologic technicians, sonographers and therapy radiographers) who completed the survey are classified as allied health professionals, per the professional categorization in the health structure of Ghana. The professionals play a key role to ensure that spread of SARS-COV-2 within the radiation medicine centres is at the barest minimum. Together with other health professionals such as doctors and nurses, some of these professionals who work at the major health facilities and at the COVID-19 isolation and treatment centres were classified as frontline health workers. This meant that the level of knowledge of radiation medicine professionals on the symptoms of COVID19 ought to be high. This is because good knowledge about the symptoms of COVID-19 has been identified as one of the key elements in winning the war against the pandemic [9].

\subsection{Level of knowledge on the symptoms of COVID-19}

The study has identified adequate level of knowledge amongst the allied radiation medicine professionals (Table 2). The WAI of 3.8 implies that the professionals are adequately informed about the overall symptoms (common and less common as specified by the WHO) associated with COVID-19. This observation projects a high tendency of the identification of potential COVID-19 patients within the radiation medicine sector, where the professionals interact

Table 5 Plurality of perception on the use of diagnostic imaging procedures in management of COVID-19

\begin{tabular}{lll}
\hline Diagnostic Imaging Procedures & WAI & Inference \\
\hline Computed tomography (CT) is the most preferred imaging modality for screening patients for COVID- & 4.2 & Positive Consensus \\
19 & 3.6 & Positive Consensus \\
Magnetic Resonance Imaging (MRI) is least likely to be used for COVID-19 diagnosis & Diverse \\
Low dose, whole lung radiation in the form of X-rays can effectively treat severe pneumonia as a result & 3.5 & 3.2 \\
$\quad$ of COVID-19 & 2.6 & Diverse \\
Ultrasound is a reliable imaging modality for screening patients for COVID-19 & 3.5 & Diverse \\
Planar X-ray is the most preferred imaging modality for screening patients for COVID-19 & Diverse \\
Overall / Composite score & & \\
\hline
\end{tabular}

$\mathrm{WAI}=$ weighted average index on a scale of 1 to 5 
among themselves, patients and sometimes patients' relatives. Such an environment could be a fertile ground for the spread of SARS-COV-2 if professionals are not at least adequately knowledgeable in identifying symptoms of the COVID-19 disease. When symptoms of the disease are picked up early in radiation medicine centres, such suspected COVID-19 patients can quickly be isolated and be given the needed attention and care while preventing any potential spread.

The study also identified that the most known symptom was shortness of breath, a condition associated with severe to critical COVID-19 cases. A large proportion of COVID19 patients in this state may experience breathing challenges and will likely be recommended for X-ray imaging or CT scan, modalities within the domain of medical imaging professionals. This observation could be a strong reason for the exhibition of very high knowledge levels on the shortness of breath by the radiation medicine professionals.

Symptoms that were identified as less known and with limited knowledge level included loss of taste, headache and sore throat, while loss of speech and movement being serious symptoms of COVID-19 were the least known. It is undesirable that some professionals did not have adequate knowledge on the common symptoms (as specified by the WHO), implying that they may not be able to apply extra caution to a potential SARS-COV-2 infected person who is at the early stage of COVID-19 and visits a radiation medicine facility for a radiological procedure. More education is required in this direction to upscale the levels of knowledge associated with the observation above. Observation of the adequate knowledge about the common symptoms of SARSCOV-2 among the allied radiation medicine professionals in this study are similar to observations reported in studies such as Kotian et al. [1] and Nkansah et al. [4], in which knowledge of health professionals on COVID-19 were assessed.

\subsection{Level of knowledge on transmission of SARS-COV-2}

Information on the transmission routes for SARS-COV-2 has been widely disseminated in digital, social and mainstream media during the era of the COVID-19 pandemic. These are anticipated to match with the levels of knowledge of the general public, more especially health professionals, whose line of duty can easily predispose them to infection by the virus. The study identified that radiation medicine professionals have adequate knowledge (Table 3 ) on the transmission of SARS-COV-2, which is a good step towards preventing spread of the disease. Specifically, almost all (99.3\%) of the respondents indicated that SARS-COV-2 infection can be transmitted through small droplets from the nose and mouth of infected persons. As has been generally communicated by WHO and by several researchers $[10,16]$ transmission of
SARS-CoV-2 is known to occur through direct or indirect routes, or by close contact with infected persons through infected secretions such as saliva and respiratory secretions or their respiratory droplets, which are expelled when an infected person coughs, sneezes, talks or sings.

Additionally, the study revealed that there were adequate levels of knowledge regarding the facts that SARS-COV-2 infection transmission could be through airborne processes, as well as through cleaning and draping of radiation emitting equipment. Also, the respondents demonstrated adequate knowledge that COVID-19 patients are most infectious when they manifest symptoms of the disease. Studies by the WHO and other agencies have suggested that respiratory secretions or droplets expelled by infected individuals can contaminate surfaces and objects, creating fomites (contaminated surfaces). SARS-COV-2 virus could be found on those surfaces for periods ranging from hours to days, depending on the ambient environment (including temperature and humidity) and the type of surface, in particular at high concentration in health care facilities where COVID-19 patients are treated $[10,17,18]$.

Transmission of SARS-COV-2 through cooked food and through faeces, which are suggested by infectious disease experts to be less likely $[9,19]$ were adequately known by the respondents of this study. Other reported transmission modes could be bloodborne, mother-to-child, and animalto-human transmission [10]. More generalized educational interventions may be necessary for radiation medicine professionals on the transmission of SARS-COV-2.

\subsection{Perception on safety practices against SARS-COV-2 infection in radiation medicine facilities}

In controlling SARS-COV-2 infections in radiation medicine facilities, perception of the personnel about recommended control measures is key to achieving the desired compliance and by extension the needed results. In the wake of the COVID-19 pandemic, many governments, including that of Ghana (acting through their ministries of health and agencies), announced several control measures and directives to deal with the disease. In radiation medicine centres and health institutions in general, examples of such measures included wearing of appropriate personal protective equipment (PPEs) by health personnel, provision of hand washing and sanitizing facilities, decontamination of the departments, reducing numbers of patients and staff at any given time to decongest the facilities and screening (temperature checking) of persons before admission into the facilities. The study identified that similar perceptions about the directives as given by the Ministry of Health towards safety practices for controlling SARS-COV-2 infections were almost 
perfectly shared among radiation medicine professionals (Table 4).

Other good infection control practices that were highly shared among the respondents included placement of radiation medicine staff on shift system, training of the personnel on the use of PPEs and maintenance of air flow in radiation medicine centres. Instituting these measures, among others, have been documented to produce a good environment that can adequately control infection of SARS-COV-2. Example of such study by Hasford et al. [20] assesses safety measures in radiation therapy departments in Africa. Further practices that ensure good infection control and which recorded at least adequate perception levels amongst the professionals were the effectiveness of face mask against SARS-COV-2 infection and keeping to a 1-m distance away from infected COVID-19 patients. These control measures are in conformity with recommendations of the WHO [10] which advises that "at all times, practice frequent hand hygiene, physical distancing from others when possible, and respiratory etiquette; avoid crowded places, close-contact settings and confined and enclosed spaces with poor ventilation; wear fabric masks when in closed, overcrowded spaces to protect others; and ensure good environmental ventilation in all closed settings and appropriate environmental cleaning and disinfection".

There have been varied opinions and practices among scientists and health professionals on the frequency with which medical equipment should be disinfected ever since the world was first hit with COVID-19. This is reflected in the responses of the study participants, where diverse perceptions are presented as to whether it is advisable to disinfect radiation medicine equipment after each working day, or only in cases where the equipment has been used on suspected COVID-19 patients. A "school of thought" is that specific medical equipment ought to have specific recommended periods (based on the usage, frequency, etc.) beyond which disinfection must necessarily take place so as to ensure that high level of hygiene is adhered to, and transmission of viruses and other pathogens are reduced. Most diverse of the perceptions is whether scanning or treatment equipment need to be avoided for up to $1 \mathrm{~h}$ upon usage for suspected COVID patients. Some clear guidelines should be given by the Ministry of Health to personnel in radiation medicine so as to influence their judgement whenever confronted with such situation.

\subsection{Plurality of perception on the use of radiation medicine procedures for managing COVID-19}

Radiation medicine procedures have played a critical role in management of the reported COVID-19 cases in hospitals. Results from this study indicate that levels of perception among radiation medicine professionals was a positive consensus (Table 5: WAI 4.2) that CT scanning is the most preferred imaging modality for screening patients for COVID-19 while MRI is least likely to be used. Ordinarily, CT scan of the thorax region would be more preferred than the other imaging modalities for critical assessment of lung conditions that may lead to shortness of breath (as in the case of severe to critical COVID19 cases) [21, 22]. Though planar X-ray imaging of the chest is also frequently used to assess lung conditions, it is comparatively limited in diagnostic information it can provide as compared to CT scans. Expectedly, most of the respondents agreed on the score that $\mathrm{CT}$ modality is the most preferred, while diverse perceptions were displayed about planar X-ray being the most preferred. Perception about the use of low dose X-rays for treating severe pneumonia arising from COVID-19 was also diverse. This is an evolving area, with not so much research data available, hence varied perceptions as observed in the study were expected. Some studies $[20,23,24]$ have shown that lowdose, whole lung radiation in the form of $\mathrm{X}$-rays can effectively treat severe pneumonia, with minimal side effects. However, it is necessary for more research work (clinical trials) to be done to confirm the findings.

The findings of this study imply that much of the educational intervention and sharing of information among medical staff by the national COVID-19 management team need to be directed more intensely at improving the levels of knowledge on symptoms of COVID-19 and transmission of SARS-COV-2 among the radiation medicine professional in Ghana. Additionally, this study encourages radiation medicine professionals to consistently update themselves of the growing development on the use of imagining procedures for managing COVID-19 as new information becomes available globally to inform their perception.

\section{Conclusion}

As part of the measures to reduce the risk of an outbreak of SARS-COV-2 infection in radiation medicine facilities, this study shows clearly that aspects of the awareness of radiation medicine professionals on the COVID-19 pandemic are only adequate, while others need critical improvement.

Acknowledgement The authors acknowledge with thanks all radiation medicine professionals who took part in the survey.

Author contributions All authors contributed to the development of the concept, preparation of survey questionnaire, analysis of survey data and manuscript writing.

Availability of data and material Yes, there is availability of data from the survey questionnaire. 


\section{Compliance with ethical standards}

Conflict of interest The authors declare that they have no conflict of interest.

Consent to participate All authors consented to participate in the study.

Consent for publication All authors consented to publication of the study.

\section{References}

1. Kotian RP, Faujdar D, Kotian SP, D'souza B. Knowledge and understanding among medical imaging professionals in India during the rapid rise of the COVID-19 pandemic. Health and Technology, May 2020. https://doi.org/10.1007/s12553-020-00437-2

2. Putrino A, Raso M, Magazzino C, Galluccio G. Coronavirus (COVID-19) in Italy: knowledge, management of patients and clinical experience of Italian dentists during the spread of contagion. BMC Oral Health. 2020;20:200. https://doi.org/10.1186/ s12903-020-01187-3.

3. Huang C, Wang Y, Li X, Ren L, Zhao J, Hu Y, et al. Clinical features of patients infected with 2019 novel coronavirus in Wuhan. China Lancet. 2020;395:497-506.

4. Nkansah C, Serwaa D, Adarkwah LA, Osei-Boakye F, Mensah K, Tetteh P, Awudu S, Apodola A. Novel coronavirus disease 2019: knowledge, practice and preparedness: a survey of healthcare workers in the Offinso-North District, Ghana. Pan African Medical Journal, Volume 35 (2): 79.16 Jun 2020, https://doi. org/10.11604/pamj.supp.2020.35.2.23644

5. Zhao D, Yao F, Wang L, Zheng L, Gao Y, Ye J et al. A comparative study on the clinical features of COVID-19 pneumonia to other pneumonias. Clinical Infectious Diseases 2020; ciaa247

6. Worldometer COVID-19 Coronavirus Pandemic Accessed online on September 1, 2020.

7. https://www.worldometers.info/coronavirus/?utm_campaign= homeAdvegas 1 ?

8. Ghana Health Service. Accessed online on September 1, 2020. https://ghanahealthservice.org/covid19/

9. Kalra MK, Homayounieh F, Arru C, Holmberg O, Vassileva J. Jul Chest CT practice and protocols for COVID-19 from radiation dose management perspective. Eur Radiol. 2020;3:1-7. https:// doi.org/10.1007/s00330-020-07034-x.

10. World Health Organization (WHO). Q\&A: How is COVID-19 transmitted? Accessed online August 23, 2020 https://www.who.int/ emergencie s/disea ses/novel-coron aviru s-2019/questionand-answers-hub/q-a-detail/q-a-how-is-covid-19-transmitted? gclid=EAIaIQobChMI_rOW596x6wIVQe3tCh2ZuwNSEAAYASA AEgLAEPD_BwE

11. World Health Organization (WHO). Transmission of SARSCoV-2: implications for infection prevention precautions. Accessed online August 23, 2020 https://www.who.int/news-room/ commentaries/detail/transmission-of-sars-cov-2-implicationsfor-infection-prevention-precautions

12. Ghinai I, McPherson TD, Hunter JC, Kirking HL, Christiansen D, Joshi K, et al. First known person-to-person transmission of severe acute respiratory syndrome coronavirus 2 (SARS-CoV-2) in the USA. Lancet. 2020;395:1137-44.
13. Chan JF-W, Yuan S, Kok K-H, To KK-W, Chu H, Yang J, A familial cluster of pneumonia associated with the, et al. novel coronavirus indicating person-to-person transmission: a study of a family cluster. Lancet. 2019;2020(395):14-23.

14. Report of the WHO-China Joint Mission on Coronavirus Disease 2019 (COVID-19) 16-24 February 2020. Geneva: World Health Organization; 2020 (available at https://www.who.int/docs/defaultsource/coronaviruse/who-china-joint-mission-on-covid-19-finalreport.pdf).

15. Liu J, Liao X, Qian S, Yuan J, Wang F, Liu Y, et al. Community Transmission of Severe Acute Respiratory Syndrome Coronavirus 2, Shenzhen, China, 2020. Emerg Infect Dis. 2020;26:1320-3.

16. Luo L, Liu D, Liao X, Wu X, Jing Q, Zheng J, et al. Modes of contact and risk of transmission in COVID-19 among close contacts (preprint). MedRxiv. 2020. https://doi.org/10.1101/2020.03.24.20042606.

17. Burke RM, Midgley CM, Dratch A, Fenstersheib M, Haupt T, Holshue M, et al. Active Monitoring of Persons Exposed to Patients with Confirmed COVID-19 - United States, JanuaryFebruary 2020. Morb Mortal Wkly Rep. 2020;69:245-6.

18. Guo Z-D, Wang Z-Y, Zhang S-F, Li X, Li L, Li C, et al. Aerosol and Surface Distribution of Severe Acute Respiratory Syndrome Coronavirus 2 in Hospital Wards, Wuhan, China, 2020. Emerging Infectious Diseases. 2020;26(7).

19. Wu S, Wang Y, Jin X, Tian J, Liu J, Mao Y. Environmental contamination by SARS-CoV-2 in a designated hospital for coronavirus disease 2019. Am J Infect Control. 2020;S0196-6553(20):30275-83.

20. Heller L, Mota CR, Greco DB. COVID-19 faecal-oral transmission: Are we asking the right questions? Science of the Total Environment. Volume 729, 10 August 2020, 138919 https://doi. org/10.1016/j.scitotenv.2020.138919

21. Kirkby C, Mackenzie M. Is low dose radiation therapy a potential treatment for COVID-19 pneumonia? Radiotherapy and Oncology, Vol 147, June 01, 2020. https://doi.org/10.1016/j.radonc. 2020.04.004

22. Godet C, Elsendoorn A, Roblot F. Benefit of CT scanning for assessing pulmonary disease in the immunodepressed patient. Diagnostic and Interventional Imaging. 2012;93(6):425-30. https:// doi.org/10.1016/j.diii.2012.04.001.

23. Pate $\mathrm{PH}$. What are the advantages of $\mathrm{CT}$ scanning over plain chest radiography in the workup of solitary pulmonary nodules? Medscape. Accessed online on August 31, 2020. https://www.medscape. com/answers/2139920-71091/what-are-the-advan tagesof-ct-scanning-over-plain-chest-radiography-in-the-workup-ofsolitary-pulmonary-nodules\#: :text=CT\%20scanning $\% 20 \mathrm{of} \%$ 20 the $\% 20$ chest,morphologic $\% 20$ features $\% 20$ of $\% 20$ various $\%$ 20lesions.

24. News Medical Life Sciences. Two clinical trials use low-dose X-rays to treat COVID-19 pneumonia. Accessed online on August 24 2020. https://www.news-medical.net/news/20200617/Two-clinicaltrials-use-low-dose-X-rays-to-treat-COVID-19-pneumonia. aspx

25. Kefayat A, Ghahremani F. Low dose radiation therapy for COVID-19 pneumonia: A double-edged sword. Radiother Oncol. 2020;147:224-5. https://doi.org/10.1016/j.radonc.2020.04.026. 